\title{
Identification of Genes Involved in Root Growth Inhibition Under Lead Stress by Transcriptome Profiling in Arabidopsis
}

\author{
Shuangshuang Zheng ${ }^{1} \cdot$ Panrong Ren ${ }^{2} \cdot$ Mingtong Zhai ${ }^{1} \cdot$ Chuanyou $\mathrm{Li}^{2} \cdot$ Qian Chen $^{1}$ (D)
}

Published online: 14 July 2020

(C) The Author(s) 2020

\begin{abstract}
Lead $(\mathrm{Pb})$ is a heavy metal with high toxicity to plants. Root is the major organ to respond to $\mathrm{Pb}$ stress. However, little is known about how plant roots perceive $\mathrm{Pb}$ stress signaling. Here, we describe the transcriptome of Arabidopsis root tips under $\mathrm{Pb}$ stress using the RNA-seq assay. A total of 703 differentially expressed genes (DEGs) were identified and expressed at every time points. Some early-responsive DEGs $(1 \mathrm{~h})$ were predicted to be negatively involved in cell elongation and cell expansion, while some late-responsive DEGs $(24 \mathrm{~h})$ positively participated in defense of oxidative stress. Hydrogen peroxide $\left(\mathrm{H}_{2} \mathrm{O}_{2}\right)$ and superoxide $\left(\mathrm{O}_{2}{ }^{-}\right)$were increased significantly in root tips under Pb stress. Cell wall extension related gene XYLOGLUCAN ENDOTRANSGLUCOSYLASE/HYDROLASE 18 (XTH18) was induced in root tips, and xth18 showed reduced root growth inhibition by $\mathrm{Pb}$ stress. Our results revealed the potential mechanism of root growth inhibition by $\mathrm{Pb}$ stress and shed light for the further study.
\end{abstract}

Keywords Lead $(\mathrm{Pb})$ stress $\cdot$ Root growth $\cdot$ RNA-seq $\cdot$ Cell wall modification $\cdot$ XTH18

\section{Introduction}

Lead $(\mathrm{Pb})$, a heavy metal with high toxicity to plants, is regarded as the first one smelted by humans (Kalisińska

Shuangshuang Zheng, Panrong Ren and Mingtong Zhai contributed equally to this work.

Key Message Lead inhibits root growth. The early- and late-responsive genes involved in lead stress were identified by RNA-seq analysis with Arabidopsis root tips. XTH18 which is a component in cell wall modification was upregulated, and root growth of $x$ th 18 showed reduced sensitivity to lead stress, suggesting that cell wall modification is involved in root growth inhibition under lead stress.

Electronic supplementary material The online version of this article (https://doi.org/10.1007/s11105-020-01233-y) contains supplementary material, which is available to authorized users.

Chuanyou Li

cyli@genetics.ac.cn

$\triangle$ Qian Chen

chenqiangenetics@163.com

1 State Key Laboratory of Crop Biology, College of Agronomy, Shandong Agricultural University, Taian 271018, Shandong, China

2 State Key Laboratory of Plant Genomics, National Centre for Plant Gene Research, Institute of Genetics and Developmental Biology, Chinese Academy of Sciences, Beijing 100101, China
2019). In daily life, $\mathrm{Pb}$ is everywhere because of its corrosion resistance and plasticity, such as fertilizers, pesticides, and pigments (Hadi and Aziz 2015). The contamination of soil by $\mathrm{Pb}$ is mainly caused by mining, chimneys of factories using $\mathrm{Pb}$, the storage battery, and smelting. In accordance with ATSDR (Agency for Toxic Substances and Disease Registry) Priority Substance List published in 2019, $\mathrm{Pb}$ was labeled as the second most dangerous environment poison due to its toxicity (https:// www.atsdr.cdc.gov/SPL/index.html\#2019spl). In terms of plants, $\mathrm{Pb}$ is not essential for plants' growth and development, but $\mathrm{Pb}$ toxicity sharply occurs even in very low concentration. $\mathrm{Pb}$ toxicity brings morpho-physiological and biochemical changes and disturbs normal functioning of the plants from cell to organ level (Ashraf and Tang 2017).

Roots, the first line of defense against $\mathrm{Pb}$ stress, secrete exudates into the rhizosphere to chelate $\mathrm{Pb}$ and prevent their uptake into root cells. In addition, roots also uptake large amounts of $\mathrm{Pb}$ through the NRAMP (natural resistanceassociated macrophage protein), ZIP (Zrt/IRT-like protein), and cation transporters, and immensely restrict its translocation to aerial parts. Simultaneously, $\mathrm{Pb}$ particles can be fixed on cell wall by extracellular carbohydrates such as callose and mucilage. It is found that callose synthesis could be induced by $\mathrm{Pb}$ stress, which formed an efficient barrier for $\mathrm{Pb}$ penetration (Samardakiewicz et al. 2012). In cytoplasm, the 
compartmentalization and chelation of $\mathrm{Pb}$ proceeds with a faster kinetics to preclude their participation in toxic reactions. Nevertheless, the burst of reactive oxygen species (ROS) attributed to $\mathrm{Pb}$ toxicity results in cell damage and reduced root length. To confront oxidative stress, antioxidant system was activated, including non-protein thiols (cysteine and glutathione), ascorbic acid, proline, as well as antioxidant enzymes, such as superoxide dismutase (SOD), guaiacol peroxidase (GPX), ascorbate peroxidase (APX), glutathione reductase (GR), and catalase (CAT).

In this study, root tips of Arabidopsis treated without and with $\mathrm{Pb}$ stress for different time points were used for RNA-seq assays. Root growth under $\mathrm{Pb}$ stress was apparently reduced, and reactive oxygen species (ROS) was significantly increased by $\mathrm{Pb}$ stress in root tips. RNA-seq data showed that a total of 703 differentially expressed genes (DEGs) were expressed at all the time points and mainly implicated in transporter activity, ion binding, catalytic activity, plant growth and development, cell wall modification, and abiotic stimulus. Further results demonstrated that the cell wall extension related gene XTH18 was up-regulated by $\mathrm{Pb}$ and $x$ th 18 showed reduced sensitivity to $\mathrm{Pb}$ stress. These results will enrich our understanding of how plant roots perceive $\mathrm{Pb}$ stress signaling and provide a solid foundation for breeding of $\mathrm{Pb}$-tolerant crop varieties.

\section{Materials and Methods}

\section{Plant Material and Root Imaging}

The Arabidopsis wild genotype (Columbia ecotype, Col-0) was served as experimental materials in the current study. Seeds were surface-sterilized in $10 \%$ bleach for $10 \mathrm{~min}$ and washed 6 times with double-distilled water to remove residual bleach. The pre-treated seeds were plated on half-strength Murashige and Skoog (MS) medium. Plants were stratified at $4{ }^{\circ} \mathrm{C}$ for 2 days in darkness and then transferred to a phytotrone set at $22^{\circ} \mathrm{C}$ with a 16 -h-light/8-h-dark photoperiod (light intensity $100 \mu \mathrm{mol} \mathrm{m} \mathrm{m}^{-2} \mathrm{~s}^{-1}$ ), which were vertically placed in a plant incubator $\left(22{ }^{\circ} \mathrm{C}\right.$, day/night). The ImageJ was applied to measure root length of 6-day-old seedlings from images captured with a Canon EOS 760D camera. The assay had three biological replicates with ten seedlings per replicate.

\section{Meristem Size Measurements and GUS Staining}

The length of root meristem was measured on a ZEISS Axio Imager Z2 microscope using six-day-old seedlings treated with or without $1 \mathrm{mM} \mathrm{Pb}$. Histochemical GUS staining was performed as described previously (Zhang et al. 2018). These experiments were replicated three times for each of ten seedlings.

\section{RNA-Seq Assay}

Five-day-old wild-type Seedlings cultured on 1/2 MS medium were transferred to medium supplemented with $1 \mathrm{mM}$ $\mathrm{Pb}\left(\mathrm{NO}_{3}\right)_{2}$ for $1 \mathrm{~h}, 6 \mathrm{~h}, 12 \mathrm{~h}$, and $24 \mathrm{~h}$. Root tips $(0.5 \mathrm{~cm})$ were collected and quickly frozen with liquid nitrogen, and seedlings on 1/2 MS medium were used as control. Total RNA was isolated using TRIzol reagent (Invitrogen, 15596026). Sequencing libraries were constructed with a total of $3 \mu \mathrm{g}$ RNA and implemented on Illumina HiSeq 2500 platform (Berry Genomics). All samples were run in three biological replicates. The Kallisto (v 0.44.0) was applied to quantify the transcripts expression levels for each read. The HISAT2 (v 2.0.5) was used to map reads to the Arabidopsis genome. DEGs were analyzed with the DESeq2 package, and genes $\left(P_{\text {adj }}<0.01\right.$ and $\mid \log _{2}$ FoldChange $\left.\mid>0.1\right)$ of which were regarded as the significant DEGs and picked for gene ontology (GO) enrichment analysis.

\section{Reverse Transcription Quantitative PCR}

Reverse transcription quantitative PCR (RT-qPCR) assays were performed to validate the dependability of RNA-seq. All RT-qPCR analyses were conducted with three biological replicates and 3 technical replicates within each biological replicate on a Bio-RAD CFX96 $6^{\mathrm{TM}}$ optics module. The relative expression level was computed by the method of comparative $\triangle \mathrm{Ct}$, and Actin7 (At5g09810) was picked to normalize all results. Specific primers are listed in Supplementary Table 1.

\section{Histochemical Detection of $\mathrm{O}_{2}^{-}$and $\mathrm{H}_{2} \mathrm{O}_{2}$ in Root}

The 4-day-old seedlings were transferred to the medium with or without $1 \mathrm{mM} \mathrm{Pb}\left(\mathrm{NO}_{3}\right)_{2}$ for $24 \mathrm{~h}$. The seedlings were immersed in 3,3'-diaminobenzidine (DAB) or nitroblue tetraxolium (NBT) staining solution for detection of $\mathrm{H}_{2} \mathrm{O}_{2}$ or $\mathrm{O}_{2}{ }^{-}$in light of the methods described in the former study, respectively (Dunand et al. 2007). Three biological replicates with ten seedlings per replicate.

\section{Results}

\section{Root Growth Inhibition by Pb Stress}

In this study, seedlings of Col- 0 were constantly fostered with or without $1 \mathrm{mM} \mathrm{Pb}\left(\mathrm{NO}_{3}\right)_{2}$ for 6 days. The results showed that root elongation was apparently inhibited, and root length was decreased by $64.37 \%$ under $\mathrm{Pb}$ stress compared with that of mock treatment (1/2 MS), while shoot growth was 
relatively less affected (Fig. $1 \mathrm{a}$ and b). Postembryonic root growth of higher plants is maintained by the root meristem. The reduction of root meristem size by $\mathrm{Pb}$ could result from its negative effect on cell division (Fig. 1c). The $C Y C B 1 ; 1$ encodes a cyclin-dependent protein kinase and plays an important role in the G2/M phase of the cell cycle. Therefore, the expression of CYCB1;1pro: GUS in roots can be used to mark mitotic activity in meristems (Colon-Carmona et al. 1999). The CYCB1;1pro:GUS was expressed in the actively dividing cells of the root meristem, and the activity was markedly
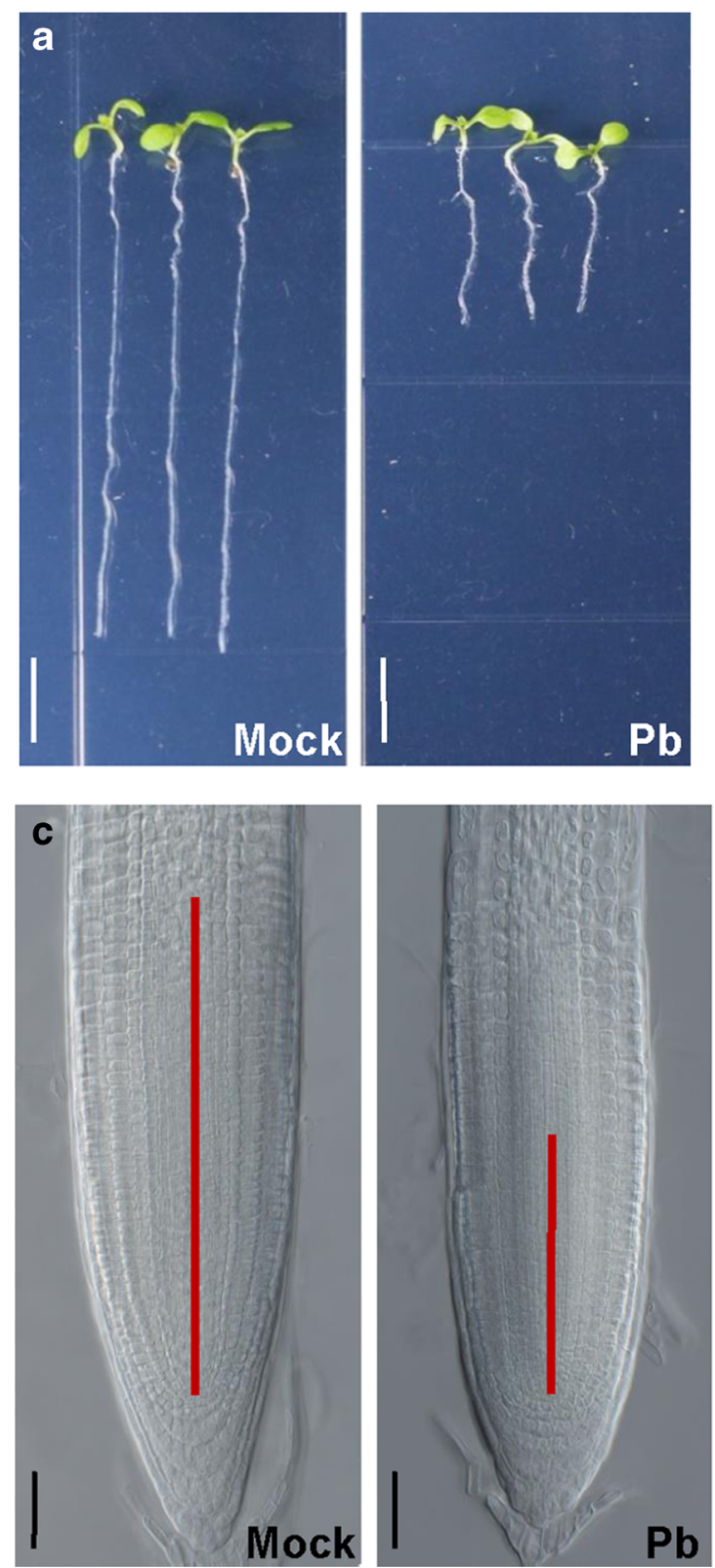

Fig. 1 Lead $(\mathrm{Pb})$ inhibits root growth. a Root length was greatly reduced by $\mathrm{Pb}$ stress. Seedlings were grown with or without $1 \mathrm{mM} \mathrm{Pb}$ for 6 days. Bar $=0.25 \mathrm{~cm}$. b Comparison of root length under the Mock (left) and $\mathrm{Pb}$ stress (right) condition. Data are shown as the average $( \pm \mathrm{SD})(n=10)$. $\mathbf{c}$ The root meristem size decreased sharply under Pb stress. Bar $=50 \mu \mathrm{m}$. d reduced by $\mathrm{Pb}$ stress (Fig. 1d), suggesting that $\mathrm{Pb}$ stress represses the cell division activity of the transit amplifying cells in the root meristem.

\section{RNA Sequencing and Identification of $\mathrm{DEGs}$ Under $\mathrm{Pb}$ Stress}

To further explore the underlying mechanism of root growth inhibition in response to $\mathrm{Pb}$ stress, RNA-seq assays were carried out with $\mathrm{Pb}$ treatments for different time points $(0 \mathrm{~h} / 1 \mathrm{~h} /$
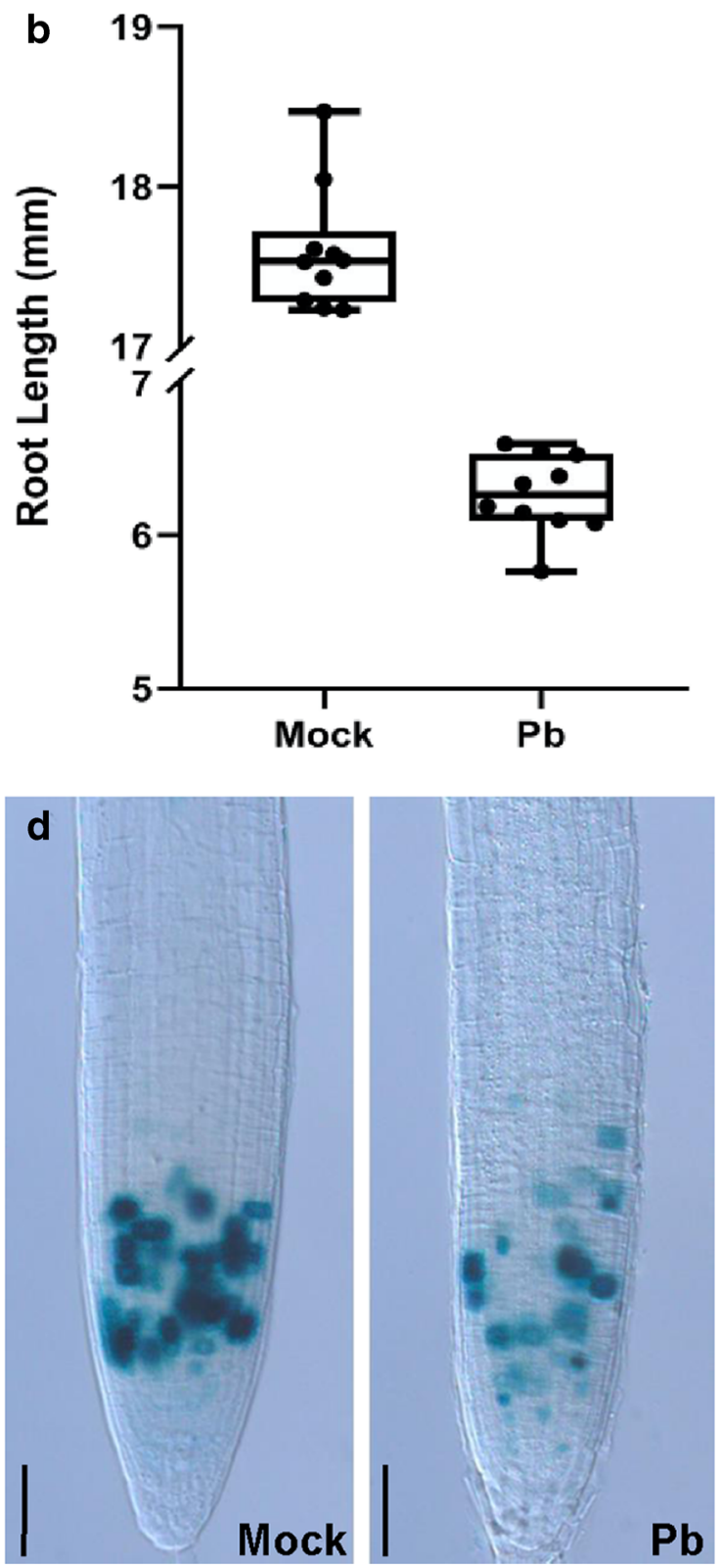

Six-day-old transgenic Arabidopsis seedlings expressing proCYCB1;1:GUS were germinated on Murashige and Skoog (MS) medium and transferred to medium without $\mathrm{Pb}$ (MS, Mock) or medium containing $1 \mathrm{mM} \mathrm{Pb}$ for $24 \mathrm{~h}$, and proCYCB1;1:GUS expression was monitored by histochemical GUS staining. Bar $=50 \mu \mathrm{m}$ 
$6 \mathrm{~h} / 12 \mathrm{~h} / 24 \mathrm{~h})$. To test the repeatability and reliability of the sequencing data, the principal component analysis (PCA) was implemented and the results demonstrated that all samples could be divided into five groups (Supplementary Fig. 1), representing that the datasets were available for further analysis. Statistically, a total of 443,708,633 raw reads were obtained from RNA sequencing. The amount of clean reads ranged from $22,626,671$ to $33,541,052$ among 15 root samples, of which between $78.93 \%$ and $94.569 \%$ mapped to the Arabidopsis genome (Supplemental Table 2). The reads uniquely mapped to the genome varies between 61.37 and $85.09 \%$. In addition, the minimum Q20 and Q30 of those clean reads were $99.86 \%$ and $82.77 \%$, respectively.

The dataset has been uploaded to NCBI database (https:// dataview.ncbi.nlm.nih.gov/object/PRJNA593333?reviewer= 422k02ihk6skl0tphuhj2oml2r).

Identification and quantification of genes (especially DEGs) expressed under the specific conditions are two vital characters of transcriptome sequencing analysis. In this study, DEGs in response to $\mathrm{Pb}$ stress were screened with the threshold of adjusted $P$ value $<0.01$. A total of 4284 DEGs were identified at $1 \mathrm{~h}$ after $\mathrm{Pb}$ stress. With $\mathrm{Pb}$ treatments from 0 to $24 \mathrm{~h}$, the number of DEGs reached a peak at $6 \mathrm{~h}$. The up- and down-regulated DGEs reached 2358 and 2739, respectively. However, the amount of DEGs sharply dropped to the minimum value at $12 \mathrm{~h}$. With $\mathrm{Pb}$ treatment for $24 \mathrm{~h}$, the amount of up-regulated DEGs was slightly more than that of down-regulated DEGs, and the total number of DEGs was 3498 (Fig. 2a and Supplementary Table 3). Subsequently, a total of 703 expressed DEGs were identified at all the time intervals (Fig. 2b). Hierarchical clustering analysis of 703 DEGs indicated that they could be divided into five groups, and the expression pattern of which was largely different. The result of cluster analyses among four groups with $\mathrm{Pb}$ treatments showed that the expression pattern of DEGs at $12 \mathrm{~h}$ was similar with that of $24 \mathrm{~h}$, and the difference of which between $\mathrm{Pb}-1 \mathrm{~h}$ and other groups was quite distinct (Fig. 2c). In addition, individual comparisons with a loop design at temporal scale were performed. A total of 3448 , 1127, 996, and 3893 DEGs were identified at 1-6 h, 6$12 \mathrm{~h}, 12-24 \mathrm{~h}$, and 1-24 h, respectively (Supplementary Table 6). For validation of RNA-seq data, a total of 14 DEGs were picked for RT-qPCR assay, and the results of correlation analysis referred that correlation coefficient between RNA-seq and RT-qPCR is 0.751 , indicating that the expression trend is consistent (Supplementary Fig. 2).

\section{GO Enrichment Analysis of Commonly Expressed DEGs}

RNA-seq provides a quick and feasible method for gene function study. Furthermore, the functional relevance of candidate genes could be analyzed with GO tools to separate gene products into three categories, including molecular function (MF), biological process (BP), and cellular component (CC). Here, the commonly expressed DEGs were also divided into three groups (Supplementary Table 4). In terms of molecular function, the processes represented by the GO terms "oxidoreductase activity", "transmembrane transporter activity," "metal ion binding," and "lipid binding" were significantly enriched (Supplementary Fig. 3), indicating that a variety of detoxification metabolisms were activated after $\mathrm{Pb}$ stress for few hours. In the biological process ontology (Supplementary Fig. 4), the major terms were "developmental growth involved in morphogenesis," "root hair cell development," "cell wall organization or biogenesis," and "response to abiotic stimulus," suggesting that plant growth (especially root development) was affected by $\mathrm{Pb}$ stress and genes related to abiotic stimulus were activated. Regarding cellular component (Supplementary Fig. 5), the major terms were "plasma membrane," "vacuolar membrane," "cell wall," and "endomembrane system," demonstrating that cell membrane system performed vital roles in $\mathrm{Pb}$ transport, chelation, and sequestration. Although many GO terms related to $\mathrm{Pb}$ stress were enriched, it still needs more information to clarify their relationship in further study.

\section{Identification of Early- and Late-Responsive Genes Under Pb Stress}

To investigate the early- and late-responsive genes related to $\mathrm{Pb}$ defense, $\mathrm{GO}$ enrichment analysis of DEGs at $1 \mathrm{~h}$ and $24 \mathrm{~h}$ was performed, and the top $20 \mathrm{GO}$ items (biological process) are shown in Supplementary Figs. 6 and 7, respectively. Interestingly, the terms named "regulation of cell size" and "cell growth" were specifically enriched at $1 \mathrm{~h}$. This result suggests that the DEGs at the early stage $(1 \mathrm{~h})$ of $\mathrm{Pb}$ stress were negatively involved in cell elongation/expansion and root growth, which demonstrated that cell elongation inhibition in root is a fast response to $\mathrm{Pb}$ stress. Furthermore, "root development," "ion transport," and "response to abiotic stress" related genes were significantly enriched at 1-6 h (Supplemental Fig. 8). Genes (Expansin, etc.) related to cell elongation were also isolated as DEGs and involved in root development, indicating that cell elongation consistently participated in defense responses of $\mathrm{Pb}$ stress at the early stage. At 6-12 h, "cell cycle," "cell division," and "response to metal ion" related genes were enriched, suggesting that $\mathrm{Pb}$ stress still affected cell division (Supplemental Fig. 9). Moreover, "oxidation-reduction process," "response to oxidative stress," and "response to metal ion" related genes were enriched at 12$24 \mathrm{~h}$ (Supplemental Fig. 10), demonstrating that oxidationreduction process was progressively activated by $\mathrm{Pb}$ stress. "Response to oxidative stress" and "plant-type cell wall organization" related genes were uniquely enriched at $24 \mathrm{~h}$ (Fig. 3a). Similarly, "oxidation-reduction process" and "response to 
a

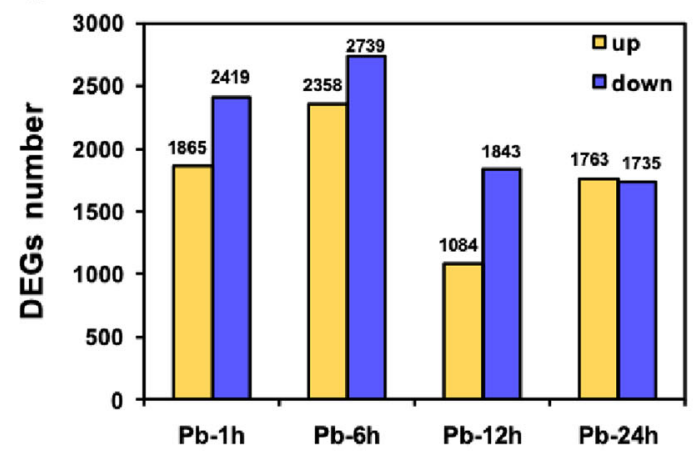

b

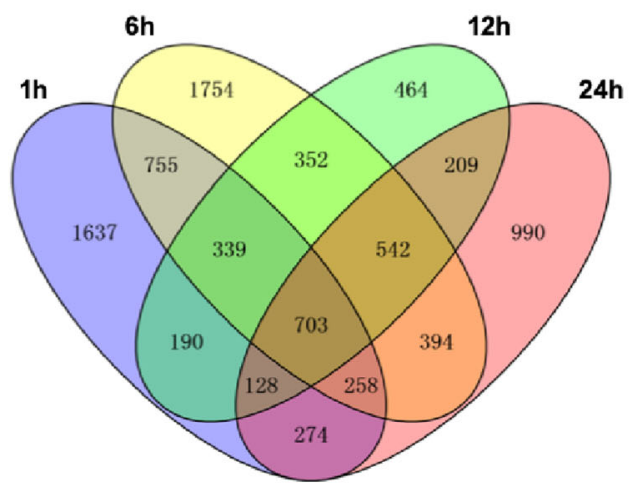

C

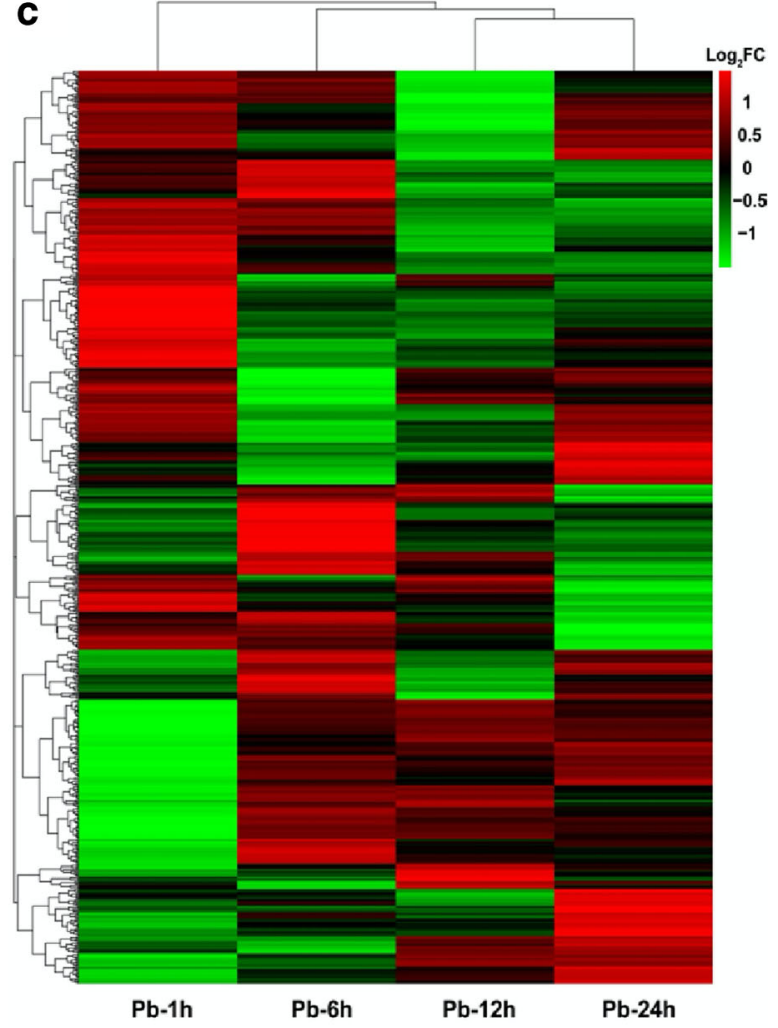

Fig. 2 Identification of DEGs and expression pattern analysis. a The number of up- and down-regulated DEGs at each time points. $\mathbf{b}$ Venn diagrams of DEGs at the indicated time points. $\mathbf{c}$ Hierarchical clustering heatmap of 703 commonly expressed DEGs

oxidative stress" related genes were also enriched at 1-24 h (Supplemental Fig. 11), implying that late-responsive DEGs were positively participated in defense against oxidative stress. Furthermore, heavy metal stress could result in the overproduction of ROS inside plant cells (Fahr et al. 2013). Accordingly, 4-day-old seedlings were used to estimate the content of hydrogen peroxide $\left(\mathrm{H}_{2} \mathrm{O}_{2}\right)$ and superoxide $\left(\mathrm{O}_{2}{ }^{-}\right)$. As shown in Fig. $3 \mathrm{~b}$ and c, the staining was significantly strengthened after $\mathrm{Pb}$ treatment for $24 \mathrm{~h}$, indicating that $\mathrm{Pb}$ indeed greatly promotes ROS production in root tips.

\section{XTH18 Plays an Important Role in Pb-Induced Root Growth Inhibition}

Xyloglucan endotransglucosylase/hydrolases (XTHs), one kind of cell wall-modifying enzymes, are correlates with hemicellulose modification and influences cellular expansion and cell wall loosening. In general, there are 33 members of the $X T H$ gene family in Arabidopsis, and 10 of them exhibited root-specific expression $(X T H-5, X T H-12, X T H-13, X T H-14$, $X T H-17, X T H-18, X T H-19, X T H-20, X T H-26$, and XTH-31). Our RNA-seq data showed that the expression of XTH5, $X T H 18, X T H 20$, and XTH31 was quickly and consistently induced by $\mathrm{Pb}$ stress (Fig. 4a). According to previous study, $X T H 18$ is also considered as one of major contributors to root growth and for normal root development (Vissenberg et al. 2005). Subsequently, we examined the effect of $\mathrm{Pb}$ on $X T H 18$ expression by expressing the GUS reporter gene under the control of the XTH18 promoter (proXTH18::GUS) in 6day-old Col-0 seedlings. The proXTH18::GUS/GFP was mainly expressed in root meristem and differentiation zone, and its expression was significantly enhanced by $\mathrm{Pb}$ treatments for $6 \mathrm{~h}, 12 \mathrm{~h}$, and $24 \mathrm{~h}$, compared with the mock $(0 \mathrm{~h})$ treatment (Fig. $4 \mathrm{~b}$ and c). We also compared $\mathrm{Pb}$-induced root growth inhibition between Col-0 (wild-type) and $x$ th 18 mutant. Root growth of $x$ th 18 showed reduced sensitivity to $\mathrm{Pb}$ stress compared with that of Col-0 (Fig. $4 \mathrm{~d}$ and e). These results indicated XTH18-mediated cell wall modification was involved in inhibition of root growth under $\mathrm{Pb}$ stress.

\section{A Putative Working Model of Pb Stress}

To better understand the molecular mechanism of plant defense against $\mathrm{Pb}$ stress, a supposed model was constructed based on our RNA-seq data (Fig. 5). Perceiving Pb stress signal was the first principle step for plants subjected to $\mathrm{Pb}$ stress. In this study, many signaling molecules were identified (Supplementary Table 5). Two calmodulin genes (At2g41110 and At3g56800) were identified and up-regulated after $\mathrm{Pb}$ stress for $1 \mathrm{~h}$, while they were constantly down-regulated at 
a

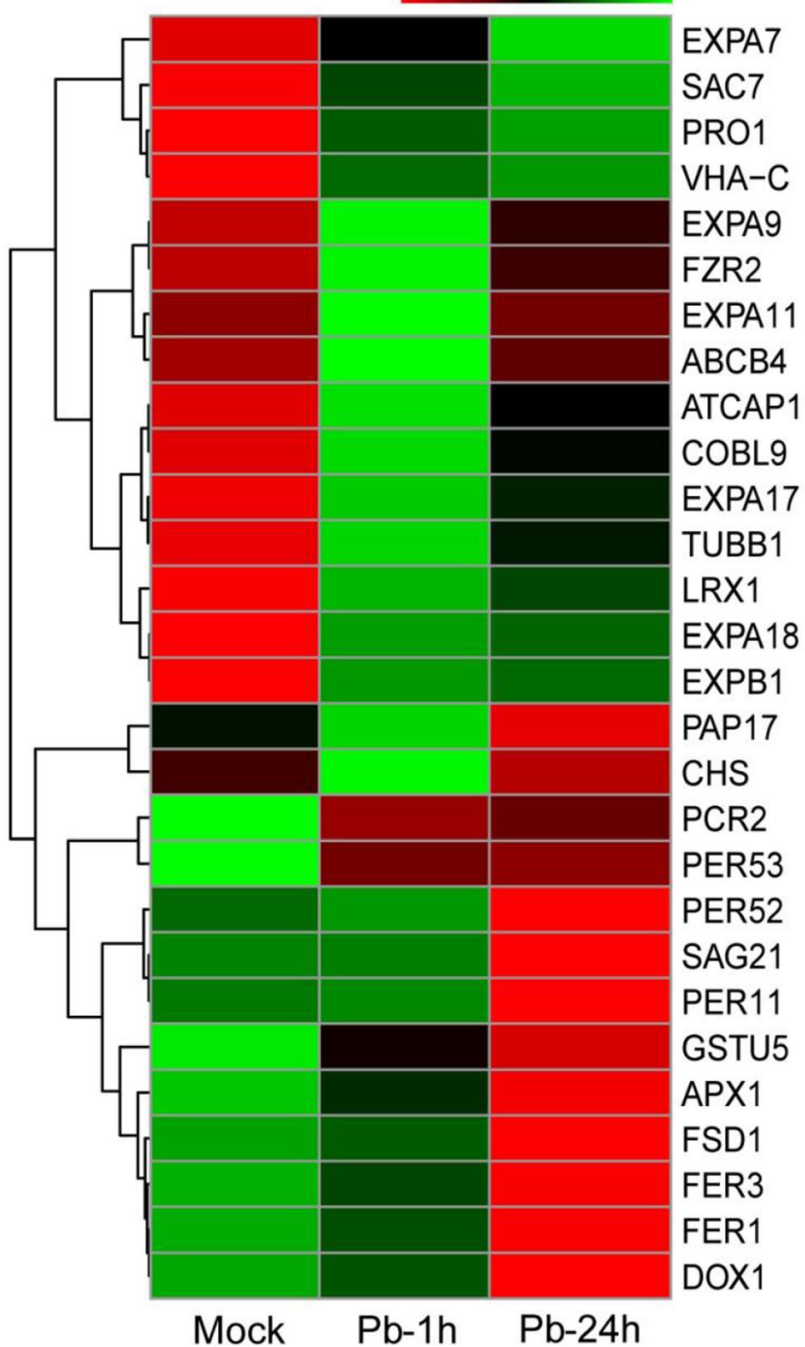

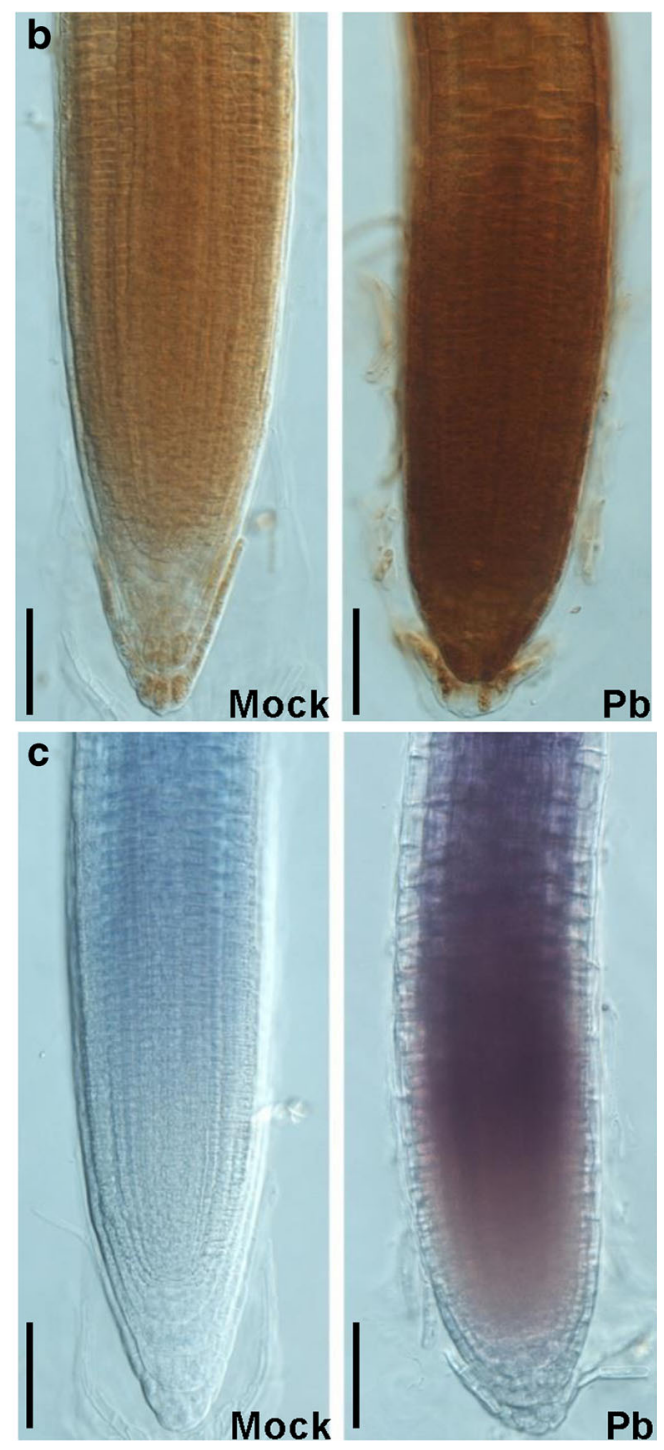

Detection of hydrogen peroxide in roots stained with DAB. Bar = $50 \mu \mathrm{m}$. $\mathrm{c}$ Detection of superoxide in roots stained with NBT. Bar $=50 \mu \mathrm{m}$
Fig. 3 Expression patterns of early- and late-responsive DEGs and physiological changes of Arabidopsis roots in response to lead $(\mathrm{Pb})$ stress. a Hierarchical clustering heatmap of partial DEGs at $1 \mathrm{~h}$ and $24 \mathrm{~h}$. b

6,12 , and $24 \mathrm{~h}$, indicating that they could be rapidly induced for responding to $\mathrm{Pb}$ stress and probably function as the sensor of $\mathrm{Pb}$ stress. In addition, three mitogen-activated protein kinase kinase kinase (MPKKK, At2g30040, At2g32510, and At4g36950) were commonly up-regulated by $\mathrm{Pb}$ stress at all time intervals and proved to be involve in stress-activated protein kinase signaling cascade in Arabidopsis. Thereafter, $\mathrm{Pb}$ was transported from rhizosphere to root and shoot through transporters. Nramp3 (At2g23150), one of the metal transporters, was induced by $\mathrm{Pb}$ stress at all time points, and expression of which reached a peak at $6 \mathrm{~h}$. We also identified three ABC transporters (At2g39350, At3g47780, and At4g01830), and two genes of which were continuously induced by $\mathrm{Pb}$. Simultaneously, $\mathrm{Pb}$ particles could be absorbed by cell wall. In the cytoplasm, the majority of $\mathrm{Pb}$ ion, with or without chelation by phytochelatins, were finally compartmented in vacuole. One cation $/ \mathrm{H}^{+}$antiporter (At5g41610) was isolated and upregulated at all time points except stress for $12 \mathrm{~h}$. Meanwhile, the increased $\mathrm{Pb}$ in the cell was responsible for generation of ROS (Ashraf et al. 2017). Under $\mathrm{Pb}$ stress condition, a large deal of ROS was generated in chloroplast by photosynthetic system and mitochondrion by respiratory electron transport chain (RETC). Three DEGs encoding NADPH related reductase (At 1 g10310, At2g17420, and At4g30210) were isolated, while expressed pattern of which was different under Pb stress. Two nitrate reductase (NADH, At 1 g37130 and At1g77760) were consistently induced by $\mathrm{Pb}$ stress, and their expression level reached peaks at $1 \mathrm{~h}$. Furthermore, ROS were also generated through respiratory burst oxidase homolog $(\mathrm{RBOH})$ located on cell 
a

$\begin{array}{lllll}1 & 0.5 & 0 & -0.5 & -1\end{array}$

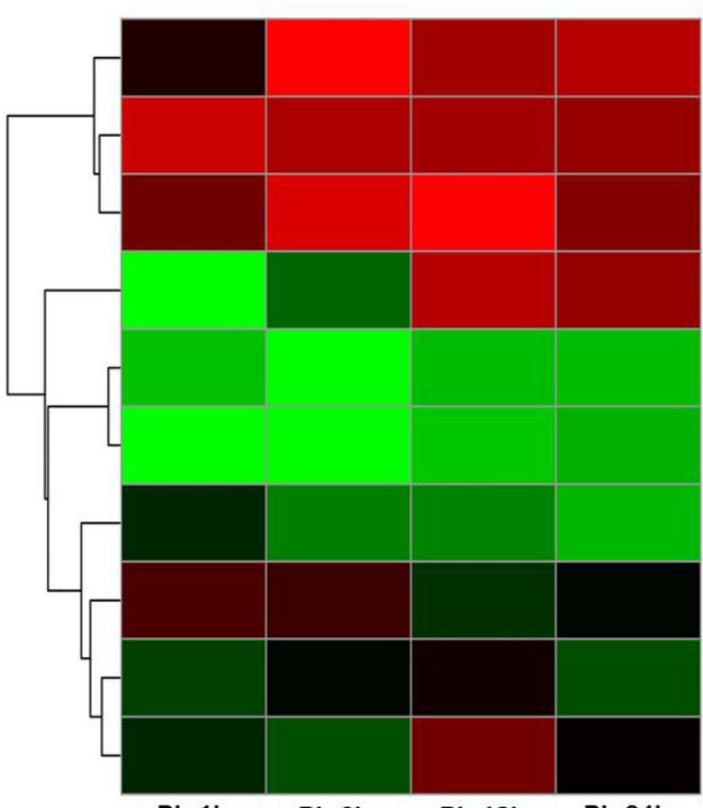

$\begin{array}{llll}\text { Pb-1h } & \text { Pb-6h } & \text { Pb-12h } & \text { Pb-24h }\end{array}$

d
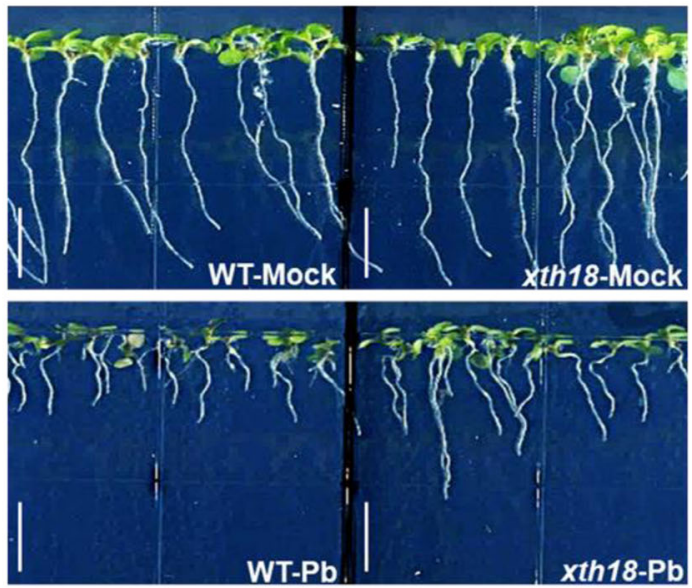

b

XTH2O

XTH18

XTH17

XTH31

$X T H 12$

XTH13

XTH17

XTH19

XTH14

XTH26

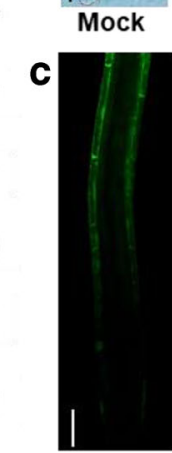

Mock
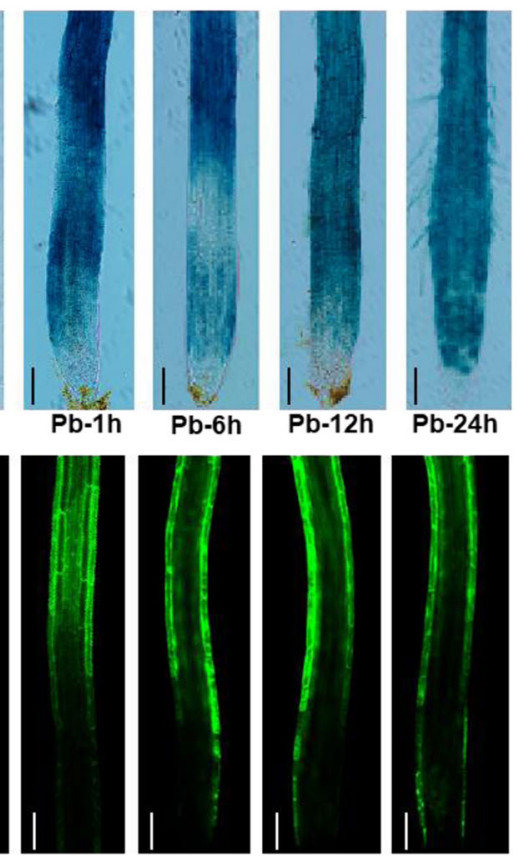

e

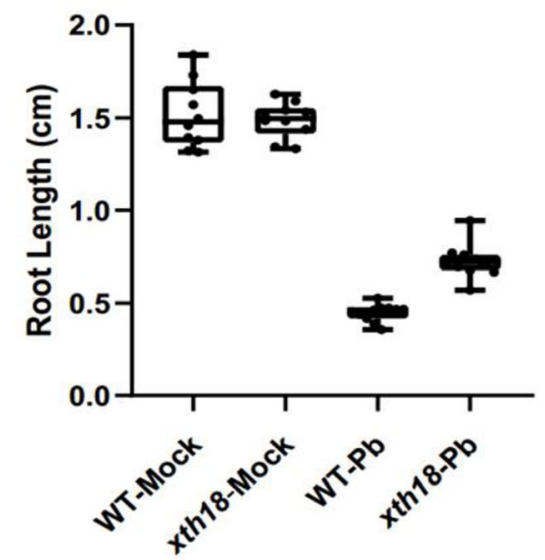

Fig. 4 Phenotype of the $x$ th 18 mutant and the expression pattern of $X T H 18$ under mock and $\mathrm{Pb}$ stress. a Heatmap of ten root-specific expressed $X T H S$ at four time points. b, c Six-day-old transgenic proXTH18::GUS/GFP seedlings were exposed to 0 (Mock) and $1 \mathrm{mM}$

membrane. To balance the cell metabolism, the activity of oxidoreductases was enhanced to scavenge ROS. Two genes encoding SOD (At2g28190 and At5g23310) were rapidly upregulated at $1 \mathrm{~h}$ and their expression level reach peak after $\mathrm{Pb}$ stress for $12 \mathrm{~h}$. A protective enzyme POD (At4g08770) was also induced by $\mathrm{Pb}$ stress at all time points. Genes encoding CAT (At4g35090) and peroxidase (At2g48150) were upregulated under $\mathrm{Pb}$ stress to transform hydrogen peroxide to water. As shown in Supplemental Figs. 12 and 13, genes involved in $\mathrm{Pb}$ stress were differentially expressed and their correlation coefficient between RNA-seq and RT-qPCR is 0.805 .
$\mathrm{Pb}$ for 6 h, 12 h, and 24 h. Bar $=100 \mu \mathrm{m}$. d, e Root growth of wild-type and $x$ th 18 plants after 6-day exposure to 0 (Mock) and $1 \mathrm{mM} \mathrm{Pb}$. Data are shown as the average $( \pm \mathrm{SD})(n=10) . \mathrm{Bar}=0.5 \mathrm{~cm}$

Meanwhile, the ROS signaling pathway was activated to stimulate the defense responses.

\section{Discussion}

Over the years, heavy metal contaminants attributed to anthropogenic activities are becoming more and more severe. As a nonessential element for plant growth and development, the release of $\mathrm{Pb}$ into environment results in declined crop yield and threatens human health. Accordingly, it is urgent to understand the underlying molecular mechanism of $\mathrm{Pb}$ tolerance 


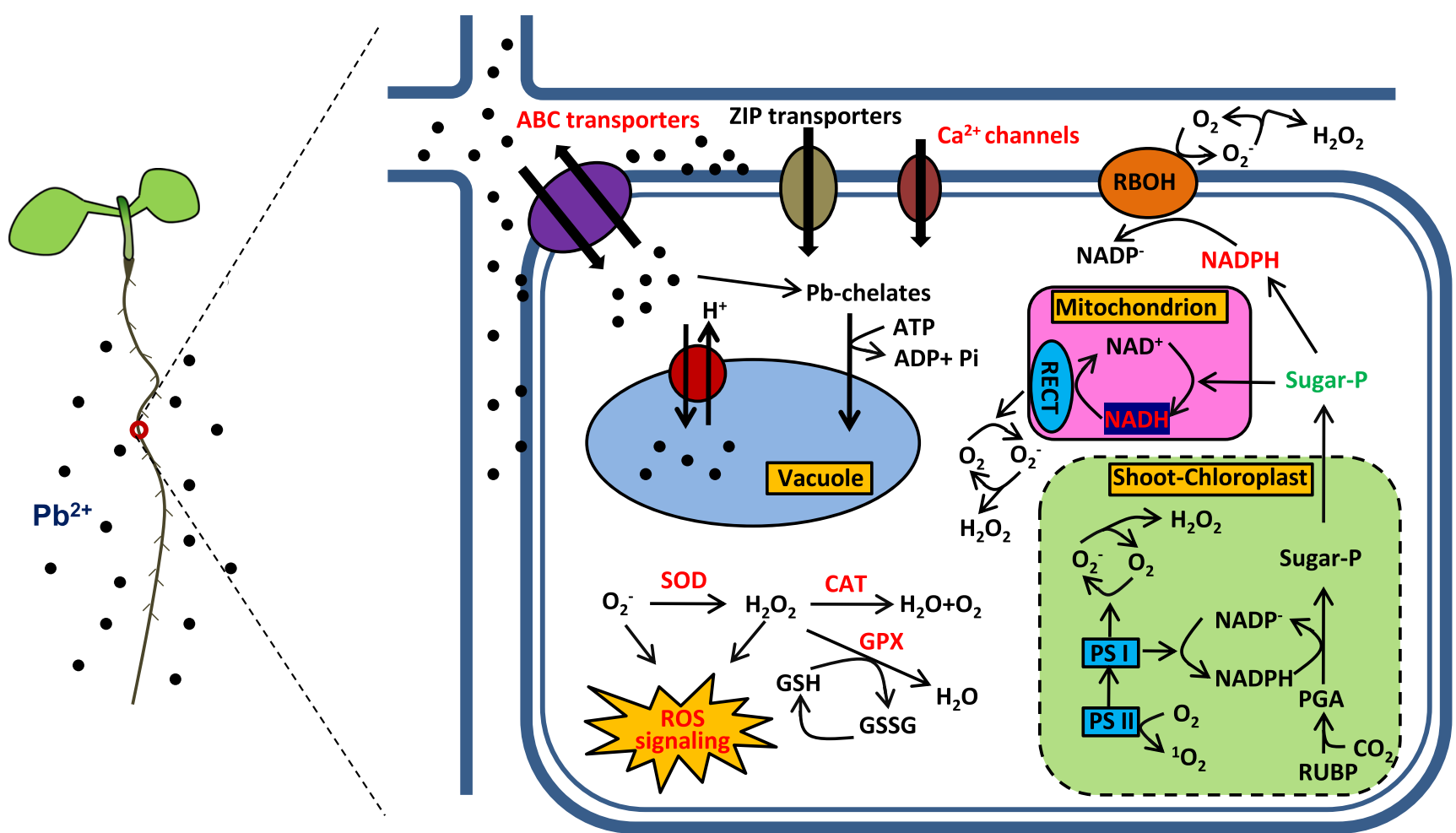

Fig. 5 Models of molecular mechanism against $\mathrm{Pb}$ stress in Arabidopsis root. Under $\mathrm{Pb}$ stress, the root growth was drastically inhibited. Subsequently, $\mathrm{Pb}$ particles were transported from rhizosphere into root, and lots of which were absorbed on cell wall or transferred into cell through membrane transporters (such as ABC transporters, ZIP transporters, and $\mathrm{Ca}^{2+}$ channels). While some of them were still stuck in the intercellular space. In the cytoplasm, $\mathrm{Pb}$ ion can be chelated by phytochelatins to synthesis $\mathrm{Pb}$-chelates, which reacted with ATP to release the energy for normal life activities under the certain conditions and finally stored in the vacuole. Simultaneously, the majority of unchelated $\mathrm{Pb}$ ion was pumped inside the vacuole though the membrane proton pump. In addition, $\mathrm{Pb}$ ion also could be transported from root to shoot. With the accumulation of $\mathrm{Pb}$ in plant, a large deal of reactive oxygen species (ROS) was produced and regarded as the messenger against $\mathrm{Pb}$ stress. In terms of the chloroplast in the shoot, oxygen could be transformed to superoxide ion based on the photosynthetic system. Meanwhile, sugar-P was synthesized with the reaction of PGA and $\mathrm{NADPH}$ and transported to root. Under the catalytic action of sugar-P and $\mathrm{NADPH}$, the majority of ROS was generated in intercellular space through $\mathrm{RBOH}$ and mitochondrion by respiratory electron transport chain (RECT), respectively. The ROS was transferred as the messenger to stimulate the defense responses against $\mathrm{Pb}$ stress. Subsequently, superoxide ion was transformed to hydrogen peroxide with the reaction of SOD, which was resolved into water and oxygen by antioxidant enzymes $\mathrm{CAT}$ and GPX. The black spots represent $\mathrm{Pb}$ ion. The bold curved lines with marine blue represent cell wall. The thin curved lines with marine blue represent cell membrane. The pansy oval represents $\mathrm{ABC}$ transporters. The pansy oval represents $\mathrm{ABC}$ transporters. The oval with dark khaki represents ZIP transporters. The oval with cardinal red represents $\mathrm{Ca}^{2+}$ channels. The tangerine oval represents $\mathrm{RBOH}$. The bigger oval with cornflower blue represents vacuole. The circle with crimson represents antiporter. The pink rectangle represents mitochondrion. The oval with deep sky blue represents RECT. The dotted square with green represents chloroplast in shoot. The blue rectangles represent photosynthetic system. The irregular shape with yellow and red words represents ROS signaling. The red words represent their coding genes were u-pregulated under $\mathrm{Pb}$ stress. The green words represent the coding genes were downregulated under $\mathrm{Pb}$ stress. $\mathrm{Pb}$ lead, ZIP Zrt/IRT-like protein, $\mathrm{RBOH}$ respiratory burst oxidase homolog, ATP adenosine triphosphate, ADP adenosine diphosphate, NADPH nicotinamide adenine dinucleotide phosphate, RECT respiratory electron transport chain, Sugar-P sugar phosphate, PSI/II photosystem I/II, PGA 3-phosphoglycerate, RUBP ribulose 1,5-bisphosphate, SOD superoxide dismutase, CAT catalase, GPX guaiacol peroxidase, GSH glutathione, reduced, GSSG glutathione, oxidized, ROS reactive oxygen species

is consistent with the former studies. However, the shoot growth of Col-0 was not much influenced at the same level of $\mathrm{Pb}$ stress, which might be due to the duration and concentration of $\mathrm{Pb}$ treatment. Another possible reason is that root is more sensitive than other organs in response to abiotic stresses. Cell division activity and root meristem size were greatly reduced by $\mathrm{Pb}$ stress, which implies inhibition of root growth by $\mathrm{Pb}$ stress is a complex process.

RNA-seq has been widely applied to identify the DEGs among different conditions in Arabidopsis (Loraine et al. declined under $\mathrm{Pb}$ treatment compared with the control, which 
2013). Nevertheless, there is little study on investigation of $\mathrm{Pb}$ stress by transcriptome profiling. In this study, a total of 15 samples were collected for RNA-seq analysis. According to Supplementary Table 2, the raw reads and total mapped rates of Col-Pb-0 h-3 were 22,880,621 and $78.93 \%$, respectively, which were also lower than that of two others in the same group, speculating that these different results could be attributed to substrate depletion during sequencing processes. Regarding DEGs number, the number of down-regulated genes were much more than that of up-regulated genes at $1 \mathrm{~h}, 6 \mathrm{~h}$, and $12 \mathrm{~h}$, while the contrary situation occurred at $24 \mathrm{~h}$ (Fig. 2a). The results of previous study found that the number of up-regulated DEGs at $12 \mathrm{~h}$ was less than that at $24 \mathrm{~h}$ under $\mathrm{Pb}$ stress in maize root, and the number of downregulated DEGs at $12 \mathrm{~h}$ was more than that at $24 \mathrm{~h}$, consistent with the results in our study. These results indicated that the onset of response to $\mathrm{Pb}$ stress is early and the metabolism gradually reached a steady state in vivo. Additionally, a total of 703 commonly expressed DEGs were identified at all the time intervals (Fig. 2c). GO annotation analysis is regarded as a valid way for comprehensive investigation of gene function. In the previous study, genes involved in metal ion binding (GO: 0046872) were regarded to bind the harmful metal ions for chelate synthesis (Ortiz et al. 2019). In terms of GO analysis of DEGs under Pb stress, a lot of GO terms (transporter activity, catalytic activity, developmental growth, response to abiotic stimulus, cell wall, etc.) were enriched, and most of which were also identified in the current study, demonstrating that the strategies for confronting $\mathrm{Pb}$ stress were similar among different species (Tian et al. 2015). As shown in Fig. $3 \mathrm{a}$, the expression level of partial DEGs at $1 \mathrm{~h}$ and $24 \mathrm{~h}$ was displayed and the changes were significant, which could be regarded as the marker genes at early and late stages of $\mathrm{Pb}$ stress. The accumulation of ROS resulted from heavy metal stress has been reported in previous study (Ashraf et al. 2017). As shown in Fig. $1 \mathrm{c}$ and d, the color of root was markedly deepened after $\mathrm{Pb}$ stress, suggesting that large amounts of ROS were produced, which could be an important indicator of $\mathrm{Pb}$ stress.

When plants are exposed to environmental stress conditions, cell wall functions as a barrier against stress factors including metals. Under $\mathrm{Pb}$ stress, $\mathrm{Pb}$ particles can be fixed on cell wall, which affects the cell wall loosening. XTHs correlate with hemicellulose modification and influence cellular expansion and cell wall loosening. In addition, XTH18 is considered as one of major contributors to root growth and for normal root development (Vissenberg et al. 2005). In this study, the expression of XTH18 was induced and the xth 18 mutant shows reduced sensitivity under $\mathrm{Pb}$ stress; these results indicated that cell wall modification is involved in lead stress response.

Signal transduction is a key step for plant defense system. During this process, a great quantity of signaling molecules and stress-related proteins were synthesized to activate specific genes for dealing with the stimulus. Calmodulin and mitogen-activated protein kinase kinase kinase (MAPKKK) have been recognized as essential signal molecules in response to stresses (Loraine et al. 2013). In our study, three MAPKKK genes were always up-regulated after $\mathrm{Pb}$ treatment at four time points, while two calmodulin genes were only upregulated at $1 \mathrm{~h}$ and down-regulated at $6 \mathrm{~h}, 12 \mathrm{~h}$, and $24 \mathrm{~h}$, indicating that signal transduction of stress was coordinated. According to previous study, $\mathrm{Pb}$ is likely transported by nonspecific transporters (Loraine et al. 2013). Therefore, $\mathrm{Pb}$ was absorbed into root and finally transported into vacuoles via membrane transporters, such as genes of ABC, ZIP, NRAMP, zinc transporter families, and antiporters. As shown in Supplementary Table 5, a total of 20 significant DEGs related to $\mathrm{Pb}$ transport, chelation, and sequestration were isolated for $\mathrm{Pb}$ metabolism. Pectin is considered as one of the $\mathrm{Pb}$ chelating agents, and the expression level of its lyase decreased sharply at four time points, suggesting that synthesis of pectin was activated under $\mathrm{Pb}$ stress. Krämer et al. (2007) reported that the NRAMP transporters existed in root and shoot, and played an essential role in transport of metal ions through the plasma membrane and the tonoplast (Krämer et al. 2007), which was up-regulated at four time points after $\mathrm{Pb}$ treatment. In addition, the content of ROS increased markedly when exposed to abiotic stress (Huang et al. 2019). In this study, two photosystem elements were up-regulated to form much ROS with the extension of the stress time. Two genes encoding respiratory burst oxidase homolog $(\mathrm{RBOH})$ protein were isolated and also upregulated at $6 \mathrm{~h}, 12 \mathrm{~h}$, and $24 \mathrm{~h}$. To improve the adverse circumstance attributed to ROS, a large amount of antioxidant enzymes was compounded to release of ROS, such as SOD, CAT, and APX. Furthermore, the ROS signaling was simultaneously motivated for further response to $\mathrm{Pb}$ stress.

\section{Conclusion}

In summary, our study is mainly focused on the root growth inhibition of model plant Arabidopsis in response to $\mathrm{Pb}$ stress at morphological, physiological, and molecular levels and found that the early-responsive genes and the late-responsive genes, and the content of hydrogen peroxide $\left(\mathrm{H}_{2} \mathrm{O}_{2}\right)$ and superoxide $\left(\mathrm{O}_{2}{ }^{-}\right)$increased significantly under $\mathrm{Pb}$ stress. In addition, XTH18, which encodes a xyloglucan endotransglucosylase, was induced by $\mathrm{Pb}$ and $x$ th 18 mutant seedling showed increased resistance to $\mathrm{Pb}$ stress, which indicated that cell wall modification was involved in of root growth inhibition under $\mathrm{Pb}$ stress. Additionally, a putative model was constructed for better understanding the defense mechanism in response to $\mathrm{Pb}$ toxicity. 
Acknowledgments This study was supported by the Basic Research Program of Shandong (Grant ZR2018ZC08N1), the Ministry of Agriculture-Chinese (2016ZX08009003-001-006), the National Key Research and Development Program of China (2019YFD1000300), and the Tai-Shan Scholars from the Shandong Provincial Government (tsqn20161021 and tsxk20150901).

\section{Compliance with Ethical Standards}

Compliance with Ethical Standards This article does not contain any studies with human participants or animals performed by any of the authors.

Conflict of Interest The authors declare that they have no conflicts of interest.

Open Access This article is licensed under a Creative Commons Attribution 4.0 International License, which permits use, sharing, adaptation, distribution and reproduction in any medium or format, as long as you give appropriate credit to the original author(s) and the source, provide a link to the Creative Commons licence, and indicate if changes were made. The images or other third party material in this article are included in the article's Creative Commons licence, unless indicated otherwise in a credit line to the material. If material is not included in the article's Creative Commons licence and your intended use is not permitted by statutory regulation or exceeds the permitted use, you will need to obtain permission directly from the copyright holder. To view a copy of this licence, visit http://creativecommons.org/licenses/by/4.0/.

\section{References}

Ashraf U, Tang X (2017) Yield and quality responses, plant metabolism and metal distribution pattern in aromatic rice under lead $(\mathrm{Pb})$ toxicity. Chemosphere 176:141-155

Ashraf U, Kanu AS, Deng Q, Mo Z, Pan S, Tian H, Tang X (2017) Lead $(\mathrm{Pb})$ toxicity; physio-biochemical mechanisms, grain yield, quality, and $\mathrm{Pb}$ distribution proportions in scented rice. Front Plant Sci 8:259

Colon-Carmona A, You R, Haimovitch-Gal T, Doerner P (1999) Technical advance: spatio-temporal analysis of mitotic activity with a labile cyclin-GUS fusion protein. Plant J 20:503-508

Dunand C, Crèvecoeur M, Penel C (2007) Distribution of superoxide and hydrogen peroxide in Arabidopsis root and their influence on root development: possible interaction with peroxidases. New Phytol 174:332-341

Fahr M, Laplaze L, Bendaou N, Hocher V, El Mzibri M, Bogusz D, Smouni A (2013) Effect of lead on root growth. Front Plant Sci 4: 175

Hadi F, Aziz T (2015) A mini review on lead (Pb) toxicity in plants. J Biol Life Sci 6:91-101

Huang H, Ullah F, Zhou DX, Yi M, Zhao Y (2019) Mechanisms of ROS regulation of plant development and stress responses. Front Plant Sci 10:800

Kalisińska E (2019) Mammals and birds as bioindicators of trace element contaminations in terrestrial environments: an ecotoxicological assessment of the Northern Hemisphere. Springer, Berlin

Krämer U, Talke IN, Hanikenne M (2007) Transition metal transport. FEBS Lett 581:2263-2272

Loraine AE, McCormick S, Estrada A, Patel K, Qin P (2013) RNA-seq of Arabidopsis pollen uncovers novel transcription and alternative splicing. Plant Physiol 162:1092-1109

Ortiz J, Soto J, Fuentes A, Herrera H, Meneses C, Arriagada C (2019) The endophytic fungus Chaetomium cupreum regulates expression of genes involved in the tolerance to metals and plant growth promotion in Eucalyptus globulus roots. Microorganisms 7:490

Samardakiewicz S, Krzesłowska M, Bilski H, Bartosiewicz R, Woźny A (2012) Is callose a barrier for lead ions entering Lemna minor L. root cells? Protoplasma 249:347-351

Seneviratne M, Gunaratne S, Bandara T, Weerasundara L, Rajakaruna N, Seneviratne G, Vithanage M (2016) Plant growth promotion by Bradyrhizobium japonicum under heavy metal stress. S Afr J Bot 105:19-24

Tian SQ, Gu CS, Liu LQ, Zhu XD, Zhao YH, Huang SZ (2015) Transcriptome profiling of Louisiana iris root and identification of genes involved in lead-stress response. Int J Mol Sci 16:2808728097

Vissenberg K, Kris V, Mika O, Yasue O, Ryusuke Y, Jean-Pierre V, Kazuhiko N (2005) Differential expression of AtXTH17, AtXTH18, AtXTH19 and AtXTH20 genes in Arabidopsis roots. Physiological roles in specification in cell wall construction. Plant Cell Physiol 46:192-200

Zhang XY, Zhou WK, Chen Q, Fang MM, Zheng SS, Scheres B, Li CY (2018) Mediator subunit MED31 is required for radial patterning of Arabidopsis roots. P Natl Acad Sci USA 115:5624-5633

Publisher's Note Springer Nature remains neutral with regard to jurisdictional claims in published maps and institutional affiliations. 